\title{
Formación internacional, retorno y ejercicio profesional de algunas mujeres mexicanas en la ingeniería, física y matemáticas: motivaciones, desafíos y estrategias
}

\section{Training abroad, return to Mexico and professional practice of Mexican women in the fields of engineering, physics and mathematics: motivations, challenges and strategies}

DOI: https://doi.org/10.32870/dse.v0i24.1036

\author{
Verónica Esquivel Camacho* \\ Isabel Izquierdo Campos**
}

\begin{abstract}
Resumen
En este artículo se estudió la formación académica internacional de un grupo de científicas mexicanas, su retorno al país, su incorporación y su ejercicio profesional en universidades públicas. Se analizaron sus motivaciones para elegir carreras científicas, los principales desafíos que enfrentaron en el campo de la ciencia, así como las estrategias que desplegaron como mecanismos de permanencia y avance en esos espacios de investigación. Se realizaron entrevistas semiestructuradas a científicas de las áreas de ingenierías y físico-matemáticas, pertenecientes a tres generaciones distintas, todas integrantes del Sistema Nacional de Investigadores. Entre las estrategias de avance, se identificó la habilidad para generar oportunidades de acceso a recursos y redes para su desarrollo académico y profesional.
\end{abstract}

Palabras clave: científicas - formación internacional - retorno - ejercicio profesional.

\begin{abstract}
In this article we studied the academic training abroad of a group of Mexican women scientists, their return to the country, and their incorporation to and professional practice in public universities. We analyzed their motivations to take up their scientific careers, the main challenges they faced in the field of science, as well as the strategies they deployed as mechanisms of permanence and progress in those research spaces. We conducted semi-structured interviews with female scientists in the areas of engineering and
\end{abstract}

* Maestra en Ciencias Sociales. Líneas de investigación: género, ciencia y universidad. Facultad de Estudios Superiores de Cuautla, Universidad Autónoma del Estado de Morelos. México. esquivelcamv2021@gmail.com

** Doctora en Pedagogía. SNI 1, miembro del COMIE. Líneas de investigación: Identidades académicas; formación y socialización de científicos/ as; educación y migración; inmigración de científicos/as; redes de colaboración en la ciencia; interacciones en educación y en ciencia entre Latinoamérica y la ex URSS. Profesora-investigadora, Universidad Autónoma del Estado de Morelos. México. isabel.izquierdo@uaem.mx 
physics-mathematics from three different generations, all of them members of Mexico's National Research Network. Among their strategies for advancement we identified the ability to generate opportunities for access to resources and networks for their academic and professional development.

Keywords: scientists - international training - return - professional practice.

\section{Introducción}

En México, durante las últimas décadas se identificó un crecimiento en la incorporación de mujeres a las universidades (Quintana, Blazquez, 2017). De manera paralela a este fenómeno, llama la atención que existen ciertas áreas del conocimiento en donde, pese a su incorporación masiva en la licenciatura (Victorino, Atriano, Rueda, 2014; Razo, 2008), ellas tienen escasa representación en comparación con los hombres, particularmente en las áreas científicas y de las ingenierías (García, 2005; De Garay, Del Valle, 2012; Arredondo, Vázquez, Velázquez, 2019; Izquierdo, Atristan, 2019). Y una vez que concluyen sus procesos formativos en ciencias, el número de mujeres que logra incorporarse a espacios laborales para ejercer sus profesiones también es menor (Zubieta, Marrero, 2005; Bustos, 2012; 2002).

Autoras como García (2005), Bustos (2008), Buquet et al. (2013), Izquierdo (2009), Blazquez y Fernández (2017), identificaron la existencia de una serie de obstáculos que tensionan y pueden dificultar el avance de las trayectorias formativas y laborales de las mujeres. En ocasiones su formación y su carrera profesional se empalma con los ciclos biológicos y los proyectos de vida, algunos de ellos tienen que ver con la constitución de una familia, la maternidad, la crianza o los roles de cuidado hacia otros (Quintana, Blázquez, 2017). En esta investigación nos centramos en mujeres que presentan una trayectoria académica y laboral sostenida cuya formación académica, en específico el doctorado, se realizó en espacios académicos internacionales y posteriormente retornaron al país para laborar en sus áreas de estudio. Por ello nos preguntamos: ¿Cómo hicieron las científicas para formarse en la ciencia e ingresar al campo laboral? ¿Qué o quién las motivó e impulsó? ¿Cuáles barreras tuvieron que afrontar y qué estrategias de avance implementaron para su desarrollo académico y laboral?

En este estudio se indagó el proceso de ingreso de las mujeres a carreras pertenecientes a las ciencias exactas e ingeniería, su movilidad internacional para realizar estudios de posgrado, su retorno a México y su inserción en la esfera profesional. Nos centramos particularmente en ese último trayecto porque es donde se identificó que se reduce el número de mujeres, segregándolas a lugares donde sus aportaciones y conocimientos pueden ser invisibilizados (Dides, Benavente, Morán, 2008; Arredondo, Vázquez, Velázquez, 2019).

Las mujeres que logran una alta calificación académica y profesional con la culminación de la etapa doctoral en el ámbito internacional y su incorporación al campo laboral, aun cuando se constituyan como una excepción a la regla como recurso humano altamente calificado, según 
estudios previos como los que aquí se citan, no están exentas de enfrentar una serie de barreras que en la práctica configuran el llamado techo de cristal y suelo pegajoso (Bustos, 2002; Cervera, Upegui, 2018; Camarena, Saavedra, 2018) para referirse a prácticas que obstaculizan e impiden el avance de las mujeres a mejores puestos en los espacios laborales. Sin embargo, algunas presentan rasgos distintivos que les permiten avanzar en sus trayectorias académicas con una motivación y un esfuerzo que logran sostenerse en el tiempo bajo el trazo de un horizonte definido, gestionando de manera efectiva las tensiones que se les presentan, mediante la puesta en marcha de estrategias de avance como mecanismos de permanencia y desarrollo hacia el logro de sus metas, tal y como fue el caso de las científicas que participaron en este estudio.

En la investigación nos centramos en dos ejes de análisis: el acceso de las mujeres a la formación académica de alto nivel y su ejercicio profesional en la ciencia, en las áreas del conocimiento de ingenierías y físico-matemáticas. La investigación se abocó a identificar y analizar las motivaciones y estrategias de avance que transgredieron los roles culturales de género desde el momento en que accedieron a la educación superior en el país, contrariando el imaginario social que ubica a la mujer en el estereotipo de esposa/madre como roles sociales, y que decidieron estudiar una licenciatura en áreas en las que tradicionalmente los hombres han tenido predominio, logrando su formación de posgrado en el extranjero en universidades de reconocido prestigio internacional, para posteriormente retornar al país e incorporarse como investigadoras en alguna Institución de Educación Superior (IES) o instituto de investigación público.

En cuanto a elementos conceptuales y siguiendo a Naranjo (2004; 2009), por motivación se entiende la puesta en acción (activación) que se dirige hacia un objetivo o meta (dirección). Los aspectos relevantes de la motivación que se retomaron en el estudio fueron tres: el primero tuvo que ver con las expectativas, es decir, las ideas que las científicas tuvieron y tienen sobre ellas mismas para ejecutar una acción; el valor con respecto a la importancia de sus metas; y"el componente afectivo, que incluye las consecuencias afectivo-emocionales derivadas de la realización de una tarea, así como de los resultados de éxito o fracaso académico" (Naranjo, 2009: 163).

El otro elemento vinculado al desarrollo académico y profesional de las protagonistas del estudio se refiere a las estrategias, entendidas como "un ensamble de acciones coordinadas para obtener una victoria, en el nivel interaccional y que es dinámica" (Kasterszein, 1999: 30). Para el caso de estudio, las entrevistadas desarrollaron cotidianamente interacciones en el campo académico en universidades extranjeras, así como en instituciones de educación superior en México, en espacios pertenecientes a disciplinas del conocimiento cuya presencia masculina es mayoritaria y, por tanto, han construido sus propias reglas de interacción social aparejadas estrechamente a su género, en tanto están asociadas a características físicas y habilidades sociales culturalmente asignadas a los hombres, tales como la fuerza física, la objetividad, el rigor matemático, la capacidad de liderazgo, entre otras, que se contraponen con el imaginario de mujer, 
construido de acuerdo a las pautas culturales dictadas por un sistema patriarcal que establece que la mujer no posee, en la misma medida que el hombre, las características y habilidades referidas (Buquet et al., 2013; Blazquez, 2011).

Al constreñirse a estas reglas, negociarlas o transgredirlas, las mujeres ponen en juego sus capacidades, y lo hicieron a partir de ciertas acciones o estrategias con miras a un horizonte: concluir su formación académica doctoral en una universidad del extranjero, para luego incorporarse como investigadoras en una institución en México. En esos trayectos tuvieron que plantearse ideas menos lejanas, tales como incluirse en los grupos de estudio, de trabajo-estudio o sociales, adaptarse a una cultura distinta, a un idioma, tener interacciones laborales sólo con grupos de hombres; algunas veces mantener una presencia discreta - casi imperceptible - en los espacios académicos, tender alianzas con miembros masculinos para hacerse parte de los grupos de investigación o para tener el aval de investigadores prestigiados que las proyectaran a universidades extranjeras a partir de sus contactos y vínculos laborales; o bien trasgrediendo las reglas, desarrollando estilos de trabajo propios, algunos muy aguerridos, directos $u$ ortodoxos, que finalmente las hacían 'distintas', no por ser mujeres sino por poseer cualidades que las caracterizaron como científicas.

\section{Metodología}

En la presente investigación se utilizó el enfoque cualitativo, que permitió acercarnos a las experiencias de las científicas a través de los relatos de vida (Pujadas, 1992), mediante la técnica de entrevista semiestructurada (Valles, 2002) que se realizó a cinco investigadoras, quienes respondieron el correo de invitación para participar en el estudio; la selección se hizo a partir de la revisión de padrones y convocatorias del Consejo Nacional de Ciencia y Tecnología (CONACyT).

Tabla 1. Participantes en el estudio

\begin{tabular}{|c|c|c|c|c|}
\hline Clave & $\begin{array}{l}\text { Carrera de } \\
\text { procedencia }\end{array}$ & $\begin{array}{l}\text { Universidad donde realizó } \\
\text { sus estudios de doctorado }\end{array}$ & $\begin{array}{l}\text { Edad } \\
\text { actual }\end{array}$ & $\begin{array}{l}\text { Estado } \\
\text { civil }\end{array}$ \\
\hline $\mathrm{IE}_{1}$ & $\begin{array}{l}\text { Ingeniería Quí- } \\
\text { mica }\end{array}$ & $\begin{array}{l}\text { Universidad de París XI, } \\
\text { Francia }\end{array}$ & 50 & Soltera \\
\hline $\mathrm{IE} 2$ & $\begin{array}{l}\text { Ingeniería Quí- } \\
\text { mica }\end{array}$ & $\begin{array}{l}\text { Universidad de British Co- } \\
\text { lumbia, Canadá }\end{array}$ & 48 & Casada \\
\hline $\mathrm{IE}_{3}$ & $\begin{array}{l}\text { Ingeniería en } \\
\text { Comunicación } \\
\text { y Electrónica }\end{array}$ & $\begin{array}{l}\text { Universidad de Duisburg, } \\
\text { Alemania }\end{array}$ & 69 & Soltera \\
\hline $\mathrm{IE}_{4}$ & Física & $\begin{array}{l}\text { Universidad de Bristol, Gran } \\
\text { Bretaña. }\end{array}$ & 52 & Casada \\
\hline $\mathrm{IE}_{5}$ & Física & $\begin{array}{l}\text { Universidad de Tennessee, } \\
\text { EE.UU. }\end{array}$ & 44 & Soltera \\
\hline
\end{tabular}

Fuente: elaboración propia con base en el trabajo de campo. 
Las científicas entrevistadas son profesoras investigadoras de tiempo completo (PITC) de centros e institutos de investigación, adscritas a IES públicas y pertenecen al Sistema Nacional de Investigadores (SNI) en los niveles I y II. Una de ellas es investigadora nacional emérita. Las participantes provienen de estratos sociales y lugares de origen distintos, dos del interior de la república (Zacatecas y Puebla) y tres de la Ciudad de México. Sus edades se encuentran entre los 44 y los 69 años, pertenecen a tres generaciones distintas, lo que permitió observar tres procesos relacionados entre sí: selección de carrera, acceso e incorporación a estudios doctorales en una universidad extranjera, retorno a México-incorporación laboral a instituciones de educación superior o institutos públicos nacionales desde diversos momentos históricos, lo que dio cuenta de variantes en las oportunidades y los sesgos por razón de género que se han reconfigurado en el país durante las décadas que comprenden sus trayectorias.

El artículo contiene cuatro partes. En primer lugar, se brinda un acercamiento al proceso de elección de carrera de las científicas, identificando las motivaciones y los retos a los que se enfrentaron; después se presentan las experiencias de movilidad internacional centradas en sus estudios de posgrado; posteriormente, se visibilizan las estrategias de avance en el proceso de su inserción y permanencia laboral al retornar al país; y, finalmente, se comparten las conclusiones del estudio.

\section{El proceso de elección de carrera: motivaciones y desafíos}

Se identificó que las motivaciones de las participantes para elegir una carrera científica fueron producto de sus deseos, a partir de la imagen de referentes positivos directos en su etapa de infancia y adolescencia, lo que las hizo "desear" ser como ciertos personajes que aparecieron previo y durante su formación universitaria, en algunos casos, particularmente reforzada por científicas que rompieron estereotipos de género en la ciencia y que han sido un ejemplo a seguir (García, 2016; Mendoza, 2016), tal y como podemos observar en el siguiente relato:

Recuerdo que una vez, la universidad fue sede del Congreso de Química Analítica, me gané la beca por promedio para asistir, fui y lo dio una doctora de la Facultad de Química de la UNAM ¡Me impresionó tanto la Doctora! Su claridad para explicar, su seguridad para explicar, para desenvolverse, me impresionó increíblemente su inteligencia y yo dije: "quiero ser algún día como ella" (IE1).

También apareció un componente que activó la motivación y “la curiosidad”, mismo que reforzó la presencia de un referente en la ciencia desde el ámbito familiar. La valoración de figuras que operaron como referentes positivos orientó, de acuerdo a Naranjo (2009), las acciones futuras en la vida académica de las jóvenes, es decir, les dio dirección para ejecutar la acción, estudiar una carrera científica: 
Mi padre trabajaba como investigador en un laboratorio, íbamos incluso los fines de semana a verlo a su trabajo, lo esperábamos en los jardines, y desde el jardín mi madre o nosotros, lo veíamos que estaba en el laboratorio con su bata trabajando y nos saludaba, entonces siempre tenía la curiosidad ¿Qué hará? ¿Qué estará haciendo ahí? (IE2).

Otro elemento que se observó en los relatos de todas las entrevistadas y que no siempre se visibiliza en los estudios sobre la formación de las mujeres en la ciencia, fue la seguridad en sí mismas reflejada en su elección profesional; si bien algunas de las participantes en el estudio cambiaron de línea de trabajo durante su proceso formativo (de acuerdo a las oportunidades que se les presentaron y a los nuevos intereses que surgieron a partir de la profundización en sus estudios, así como de las interacciones con otros investigadores más experimentados en el campo), no hubo duda respecto a la elección de carrera ni al área de conocimiento en la que querían formarse. Incluso, algunas debieron sortear dificultades familiares como la falta de apoyo económico para poder dedicarse a una carrera que no correspondía con las expectativas familiares. Aun con todo ello, en todas las participantes se identificó la seguridad de sus elecciones y la "capacidad de decidir" por sí mismas (Naranjo, 2009) en función de sus propios deseos y proyecciones futuras, desatendiendo mandatos sociales que construyen a la mujer bajo la tutela permanente de otros, cuestionando precisamente su capacidad de decidir y actuar. Eso se observó en todas las entrevistadas como un componente central de sus trayectorias y definitorio en el logro de sus metas académicas:

Siempre tuve inclinación hacia las ciencias. Por alguna razón era muy buena en matemáticas, en física. Cuando el profesor hacia preguntas para los alumnos y nadie quería participar o nadie quería resolver, decía: “¡! pase al pizarrón", entonces pasaba al pizarrón a resolver las cosas, me sentía capaz, era un reto, pero yo pensaba "yo puedo lograrlo" (IE4).

Para mí fue muy fácil, yo nunca tuve duda; yo iba a ingeniería química. Sin dudarlo, yo iba a entrar a ingeniería química, y ya, me inscribí y terminé mi carrera (IE1).

No me interesaba estudiar idiomas o psicología entonces, como que me quedé al otro lado, de ciencias exactas, más duras, más lógicas. En mi idea de avanzar más rápido, ingresé al poli y ya estando en el poli, a los dos años, fue que decidí que me quedaba con la parte de eléctrica, así fue como entré a la Escuela Superior de Ingeniería Mecánica y Eléctrica (IE3).

La elección no creo que fuera tan difícil, quizá más que nada pues, como que tus padres esperan algo de ti ¿no? Y mis padres, por ejemplo, siempre quisieron que yo me dedicara más a la medicina, pero afortunadamente mi madre siempre fue una mujer admirable, nos crio en un ambiente de que lo que 
tu quisieras hacer, estaba bien. Entonces, en ese sentido, fue más que nada, no dificultad, sino que, el temor a decepcionar un poco a tus padres (IE5).

Los relatos anteriores nos permitieron observar las maneras en que las participantes del estudio habían construido un imaginario de la carrera científica, así como una proyección de su futuro profesional que se dibujó aparejado a una serie de habilidades que ellas mismas describieron, como la posesión de un razonamiento lógico-matemático, por ejemplo, aunado a la socialización con figuras y referentes positivos en la ciencia, lo que consolidó su proyección de futuro en alguna disciplina científica. Además, se reconocieron elementos que visibilizaron la confianza en sí mismas y en sus acciones, pese a los escenarios adversos. Al respecto, concordamos con Guevara y Flores (2018): esas experiencias y aprendizajes de las jóvenes exitosas en la ciencia deben ser retomadas para el desarrollo de políticas públicas que incidan en el sistema educativo mexicano, tanto en el nivel de educación básica como en el nivel medio superior, para motivar e inspirar vocaciones científicas en las niñas y las jóvenes, particularmente en las ciencias exactas e ingeniería u otras disciplinas cuyo imaginario social las asigna generalmente a los hombres.

\section{Los estudios de posgrado: experiencias de movilidad internacional}

La movilidad internacional de la educación superior se estudió escasamente a partir de los años setenta y, a la fecha, ha tomado relevancia debido a la realidad global que enfrentamos y que nos sumerge en un mercado académico mucho más competitivo (Izquierdo, Cárdenas, 2019). Sin embargo, ya desde aquel momento se apreciaron dos desafíos para el estudiantado que decidía ir al extranjero: la dificultad con el idioma del país receptor y el ritmo más acelerado de estudio debido a un sistema más competitivo en aquellos países (Lomnitz, Morán, 1976):

El nivel que tienen los franceses en el doctorado es muy superior a la formación que nosotros llevábamos y era, además, muy denso [...]. Llegué a un laboratorio muy rico, de lo mejor que hay en Francia para lo que son las técnicas nucleares y la radioquímica, lo más difícil fue el primer año (IE1).

En el estudio que nos ocupa, en todos los casos, la realización del doctorado en un país y una universidad del extranjero se narró, en retrospectiva, como un proceso grato, más allá de la fuerte demanda académica y los desafíos que se enfrentaron al estudiar en un idioma distinto al propio:

Las personas del laboratorio pues son unas personas de lo mejor que te puedas imaginar, personas curiosas de que “QQuién eres?, ¿Por qué estás aquí? ¿Qué estás haciendo?". Obviamente todos tienen una educación alta, por lo mismo en el nivel cultural es más amplio, no cerrados a que son puros americanos, entonces, la integración se va dando poco a poco y en algún momento lo consideré mi segundo hogar, sí fue una experiencia muy grata para mí (IE5). 
Era un grupo pequeño, en esa ciudad de Duisburg, es una ciudad netamente industrial, productora de acero y donde al revés, viven muchos extranjeros migrantes. Los alemanes en esa época nos hacían inscribirnos en un lugar aparte, la persona con la que llegué parece que le sorprendió que yo quisiera estudiar ingeniería y le caí muy bien porque realmente me apoyó en toditito para conseguir la mejor casa, el profesor me apoyo muchísimo y el grupo de trabajo, que era pequeñito, éramos unas 15 gentes que estaban ahí en el laboratorio con el profesor, era un grupo muy integrado porque la universidad se acababa de fundar [...]. Alemania se estaba reformando en esa época todavía, estaba en los años setenta, tenía muchas cosas producto de la guerra, todavía dividida, los alemanes trataban de hacer su mejor papel, incluso en la universidad, con los extranjeros (IE3).

Todas las entrevistadas refirieron haber encontrado apoyos en los grupos de trabajo, principalmente con sus asesores/as de tesis, y con grupos de connacionales $u$ otros migrantes que se convirtieron en facilitadores para hacer su vida cotidiana menos tensionante: encontrar una casa para rentar, moverse en el transporte público y convivir con la burocracia de las propias universidades, así como para incorporarse a grupos y redes de convivencia social extramuros en las universidades. Todas conservan amistades y vínculos con aquellas personas y, dada la experiencia positiva que recibieron en cuanto al trato, se mostraron abiertas y manifestaron su deseo de apoyar a nuevas generaciones de jóvenes investigadoras para que también puedan tener experiencias de movilidad internacional.

El proceso administrativo que demandó el cumplimiento de requisitos para acceder a una beca del CONACyT para realizar sus estudios doctorales en el extranjero, apareció fácil de abordar, heterogéneo para mujeres y hombres, sin ninguna complejidad extraordinaria más allá del cumplimiento con los tiempos establecidos en las convocatorias y la presentación de todos los documentos que avalaban su pretensión de obtener una beca económica. Sin embargo, en algunos casos sí identificamos elementos que nos hicieron pensar en la existencia de ciertas barreras por razón de género, aunque no siempre son advertidas en las investigaciones que se han realizado sobre ingreso y deserción del posgrado (Hernández, Pérez, González, 2014), a las que las mujeres enfrentaron en sus carreras científicas cuando accedieron a becas para realizar estudios de doctorado. Todo ello se identificó en los procesos de selección, específicamente en las entrevistas, donde los comités institucionales evalúan y finalmente determinan si una aspirante a beca internacional será aceptada o no:

Cuando hice mi evaluación para el doctorado, me encontré con un comité conformado por cinco hombres. No me hicieron ninguna pregunta técnica o científica, todas eran personales. La principal, porque me hicieron mucho hincapié, era sobre los hijos, si deseaba tenerlos, a lo que respondí sin dudar desde mi convicción, y aseguré que no, "no era algo que me interesara y no pensaba tenerlos". Creo que ese fue mi pase [...] Pero pienso en las mujeres que dudaron al contestar o que dijeron que sí querían, desde ahí, "tache" (IE2). 
El relato anterior nos permite observar los mandatos de género que las mujeres deben cumplir desde el imaginario masculino, patriarcal y androcéntrico, que ubican a la mujer en exclusiva al cuidado de los otros, y brinda luz para visibilizar esa idea recurrente y oculta en las políticas públicas sobre los méritos académicos y aquello que constituye un "perfil social" de la mujer profesionista, que tiene que ver con la configuración de ciertas reglas o condiciones no escritas que deben cumplir para estar en las mismas condiciones que los hombres en el terreno de las ciencias exactas e ingeniería. En este caso, dicho perfil demanda de las mujeres no tener ningún compromiso más allá del trabajo científico, como aquel que supone la procreación y la crianza de los hijos. Es decir, mujer soltera, sin hijos, que construye en el imaginario social la idea de que, para dedicarse a la ciencia, los proyectos personales y de familia deben abandonarse; ello supone - sólo para las mujeres - una elección entre los deseos personales y los profesionales, que ya de entrada las desmembra y escinde. Todo lo anterior puede operar como un desaliento para que las más jóvenes ingresen y ejerzan carreras en las ciencias exactas, poniendo en evidencia la desigualdad y el desequilibrio en los órganos de evaluación del trabajo científico, donde el género puede sesgar el resultado de la elección $y$, bajo ciertas construcciones sociales en las que se encasilla a las mujeres, puede truncar o postergar posibilidades de desarrollo académico en el extranjero.

Al terminar sus estudios doctorales, algunas de las participantes en el estudio realizaron estancias posdoctorales, y todas en algún momento decidieron retornar a México. En la mayoría de los casos esa decisión tuvo que ver con el compromiso y responsabilidad con el país y con la institución que les apoyó económicamente para estudiar en el extranjero, pero también apareció fuertemente la constante "familia". En cuatro de los casos (dos de ellas se habían incorporado al campo laboral en los países receptores como posdoctorantes), y aún con las dificultades y desafíos que conocían respecto a la realidad económica y social de nuestro país, decidieron "regresar a casa":

El último año yo empiezo a trabajar y tengo un buen trabajo y todas las cosas están funcionando maravillosamente, y yo decía: "es que tengo que regresar, yo tengo un compromiso en el instituto y con CONACyT" y todo este rollo, mi familia y mi esposo siempre me dijo: "sí, vámonos, este no es nuestro país, nosotros sabemos que estuvimos aquí para esto, para tu doctorado, tenemos un plan", fue algo que decidí principalmente por el tema familiar (IE2).

Para nosotros [ella y su compañero] era muy importante el compromiso con CONACyT. No solo era una cuestión de que sentíamos esa responsabilidad moral, también, por ejemplo, nosotros nos fuimos con beca crédito, nosotros firmamos un pagaré en donde nos comprometíamos a regresar o regresar el dinero que se había invertido en nuestra formación. Entonces para nosotros era bien claro que teníamos que regresar al país (IE4). 
Ellos son más secos [la gente del país extranjero donde estudió], la aproximación con ellos me cuesta un poco más de trabajo y es cuando me planteo regresar, "regresar a casa". No tanto por la situación científica, sino más bien por extrañar el sentirme más integrada (IE5).

Para las participantes del estudio, en retrospectiva, el retorno se observó como parte de un proceso, de un ciclo que culminó exitosamente con la consecución de la meta académica, al obtener el grado de doctoras, pero especialmente por haber vivenciado el "estar" en otro país, lo que les permitió elaborar una nueva visión sobre la academia, la ciencia y sobre sí mismas. A partir de su experiencia, la decisión de retorno se expresó como necesaria. Refirieron que fueron beneficiadas con una beca internacional con apoyo nacional, se percibieron como afortunadas y con el firme compromiso de regresar a su país, con la convicción de contribuir con su trabajo científico.

\section{Inserción y permanencia laboral a su retorno a México: estrategias de avance} Las experiencias que las científicas tuvieron en su incursión en el campo laboral al retornar a México fueron diversas. Se observó que en dos de los casos, dado el momento histórico en que se dio su inserción laboral, existió una apertura de las instituciones para brindarles oportunidades laborales y de desarrollo. En la década de los ochenta, la investigadora de mayor edad narró la experiencia de una época en la que había oportunidades para quienes retornaban al país con una formación de alta calidad, incluso para quienes habían terminado su formación en el nivel de licenciatura. La realidad de las universidades en América Latina y en el país demandaba la expansión de las instituciones académicas y la apertura de nuevos centros de investigación, pese a las severas crisis económicas en dicha década (Victorino, Atriano y Rueda, 2014; Izquierdo, 2009). En ese momento histórico la competencia en este campo científico era muy poca; dado que igualmente eran muy pocos/as los/as investigadores/as en México, el país estaba necesitado de nuevos desarrollos científicos y de personal altamente calificado que deseara incorporarse a la investigación:

Me fue facilísimo en ese momento, además estaba el país ansioso, había plazas, entonces la gente estaba deseosa de que uno se quedara o retornara, no eran las condiciones que hay ahora, de que tienes que esperar cinco años y luego haces un posdoctorado y luego no sé qué, no, directamente "¿Quieres venir?", dije “Bueno, adelante" y “Quieres dar clases aquí en la facultad?", “SŚ, adelante!, ¡Sí!". “¿Te hacemos miembro de la Academia de Ingeniería?", "¡Sí!". Entonces estaba abierto porque éramos muy poquitos y había apoyo por parte del Estado para que realmente creciera el grupo de investigadoras en el país, ahorita parecería que no les interesa que crezca (IE3).

Más adelante, en los años noventa, otra de las entrevistadas que retornó al país en esa década a través del Programa de Repatriación gestionado por el CONACyT, igualmente detalló que 
encontró una realidad económica favorable a quienes concluían estudios doctorales y retornaban al país. Las ofertas laborales eran inmediatas y, en su caso, pudo elegir entre tres opciones laborales, según sus necesidades económicas y personales:

A mí me tocó la época, digamos, de bonanza del CONACyT y de México, a la par que me ofrecen trabajo en el instituto, me ofrecen trabajos en lo que fue mi Alma mater [...], podía escoger hasta en Chihuahua, o sea tenías posibilidades que ahora no tienes (IE1).

En cambio, la científica que retornó al país diez años más tarde, en el año 2000, narró la necesidad de recurrir a vínculos de su mismo campo para poder incorporarse a una institución universitaria; pese a ello, el periodo de tiempo entre la búsqueda de la oportunidad y la incorporación laboral no fue superior a tres meses, pero, a diferencia de las investigadoras que retornaron al país en los ochenta y noventa, ya no fue tan fácil. En adelante, y hasta la fecha, según lo relatado por cuatro de las entrevistadas, se han recrudecido las políticas de incorporación laboral en las IES e institutos públicos de México. Lo cual coincide con diversos estudios (García, 2005; Canales, 2011; Archundia, 2020) sobre políticas y financiamiento a la ciencia y tecnología.

Lo anterior, de acuerdo con las narrativas de este estudio, se traduce en mayores requisitos y mayor tiempo de espera, así como en la necesidad de una sobrecalificación profesional que no garantiza que los/as aspirantes a una plaza de tiempo completo de investigación finalmente la consigan, justificando la pertinencia de su estancia en México y la plaza que están ocupando o por ocupar, lo cual visibiliza condiciones de precariedad laboral y de escasas oportunidades para obtener un trabajo permanente (Reis, Cecílio, 2014; Basail, 2020).

El estudio también dio cuenta de la falta de políticas públicas de seguimiento por parte del CONACyT y el apoyo sostenido de políticas para reincorporar o, por lo menos, identificar los trabajos y aportes de quienes han sido beneficiados/as con becas en el extranjero y que, derivado de sus estancias (largas) en universidades en otros países, se han desvinculado de los grupos de trabajo existentes en México. Por tanto, al retorno, el recurso humano altamente calificado encuentra obstáculos y desventajas en su incorporación laboral que pueden generar su pérdida (fuga de cerebros), al emigrar en definitiva a otros países donde su trabajo puede ser más reconocido que en su país de origen:

Es en el extranjero donde tengo más reconocimiento y donde se abren las puertas fácilmente para el trabajo en colaboración, para cualquier tipo de negociación o discusión técnica, es sobre mantequilla (IE2).

Cuando yo ingresé a la UNAM, con un posdoc era suficiente, ahora hay gente que viene con dos, tres posdocs, diferentes laboratorios, diferentes partes del mundo, y realmente ya es muy competitivo encontrar una plaza de tiempo completo (IE4). 
Todo ello genera tensiones en quienes retornan como recurso humano altamente calificado y enfrentan grandes desafíos a su llegada a México, tanto para conseguir trabajo como en las promociones y permanencias, ya que los climas laborales, a decir de una de las entrevistadas, se han tornado cada vez más hostiles debido a la voraz competencia y la lucha constante por una oportunidad laboral:

En algún momento sí llegué a mi tope, las interacciones aquí no fueron particularmente suaves, porque el director en turno quería que yo estuviera debajo de otro investigador experimental aquí. Con el consejo de las personas que yo conocía pues, me dijeron que no, entonces pues, sí hubo un poquito de enfrentamiento, rumores, chismes, entonces, sí fue pesado [...]. Mi estancia aquí está un poquito señalada, está marcada por ese proceso de inserción que tuve aquí (IE5).

Mis proyectos no fueron aprobados en casi diez años, no me aprobaban ningún proyecto, quise renunciar, pero las circunstancias me mantuvieron aquí (IE2).

Acorde a los hallazgos de esta investigación, los desafíos que se presentan a su retorno a México tienen que ver con la falta de infraestructura y materiales de trabajo en los laboratorios a los que las investigadoras se incorporaron, ya sea en universidades o en institutos nacionales de investigación. Además de una fuerte problemática al interior de las instituciones debido a políticas escalafonarias regidas por sindicatos o los grupos de trabajo escindidos que se crean y agrupan en las instituciones (Ramos, Sieglin, Zúñiga, 2013), que las somete a un proceso de lucha por obtener una plaza. Una vez que se logra, las vuelve blanco de señalamientos por parte de quienes ya pertenecen a grupos de investigación versus aquellos/as que se han formado en el extranjero:

La oposición con los compañeros que ya estaban en la facultad, en la universidad fue terrible, ¿Por qué la contratan? Yo llevo tres años dando aquí clases, yo llevo cinco años y ni siquiera le hicieron examen para ingresar, ¿Por qué le dan las clases?, ¿Por qué le dan tiempo completo?" [...]; yo le puedo decir que hay cierta dificultad y desventaja para nosotros que veníamos del extranjero, porque a diferencia de los que ya estaban aquí, ya tenían cierto apoyo del doctor fulano o del maestro fulano y nosotros no, lo único que traíamos era nuestro papel, nuestro diploma de doctor (IE1).

Se identificaron otros desafíos laborales que no tienen que ver sólo con los contextos y las problemáticas expuestas anteriormente; estos se vinculan con la pertenencia al género, mismos que contradicen la idea de que por su propia naturaleza el mundo académico es un espacio neutro en relación con cuestiones de género y que, por tanto, está regido por condiciones de igualdad entre pares académicos. Asimismo, que la meritocracia es un camino seguro al éxito y que el reconocimiento profesional va aparejado, con independencia de si se es hombre o mujer: 
Sí creo que hay ambientes muchos más agresivos para la mujer [...] no sé si ya es mi carácter o es el hecho de que sí sientan que somos menos fáciles o, por lo menos yo, menos fácil de poder llegar a un ambiente de compadrazgo, de corrupción entre la gente, no lo sé, eso me ha costado trabajo aquí (IE3).

Los resultados del estudio contradicen la idea de que contar con una alta calificación académica, especialmente si se obtiene en el extranjero, se traducirá en la obtención de ventajas laborales, en este caso, el ingreso laboral inmediato a alguna IES nacional o instituto público de México. Aunado a ello, se induce la idea de que las mujeres que cuentan con esta alta calificación tampoco tienen una posición de ventaja frente a sus pares, ya sean mujeres u hombres formados académicamente en universidades del país. Las mujeres altamente calificadas en busca de una oportunidad de incorporación laboral en IES de México deben sortear desafíos e incluso enfrentarse a climas laborales hostiles, dado que, si bien tienen las competencias necesarias para obtener plazas al igual que sus pares varones, en algunos casos sus designaciones son cuestionadas y señaladas, por tanto, aunque se aceptan, en las interacciones diarias parece que nunca se legitiman:

Es muy frustrante, tú tienes que estar bien agarrada a la realidad y al piso porque es muy frustrante, hablábamos hace rato de las pérdidas cuando te vas al extranjero, justamente es eso, cuando tú llegas y tú ves a otra compañera, ves que, mucho después hizo sus estudios de doctorado, ella se fue abriendo paso y tú sigues batallando porque no tuviste ese camino que te abrió hacer el doctorado aquí, con un doctor aquí, estando y quedándote aquí (IE1).

También se dio cuenta de que uno de los problemas que dificulta la incorporación laboral, y que supone nuevos desafíos para las científicas que retornaron al país, fue la falta de recursos en las IES y la ruptura de vínculos laborales y académicos. Si bien se facilitó su retorno a México mediante el subprograma de Repatriación como una política pública (Izquierdo, 2009), ellas tuvieron que empezar de cero en los espacios laborales donde se incorporaron, pues no pertenecían a ningún grupo de trabajo y, cuando llegaron, buscaron por su cuenta a qué institución incorporarse; los vínculos con los que contaban en México se movilizaron de tal manera que, si bien su alta calificación académica les abrió nuevas posibilidades de trabajo, no siempre les garantizó las mejores condiciones, ni tampoco la consolidación de sus proyectos de investigación. Por otro lado, en este estudio se identificó que las fuertes demandas laborales en el sistema académico hicieron que las mujeres fueran más competitivas, igual que los varones; sin embargo, la competencia pareció volverse desleal entre ellas, pasando a una lucha encarnizada por ocupar una posición o avanzar en escalas laborales donde la presencia masculina es avasallante, eso que para los hombres aparece como una extrañeza y atipicidad, para las mujeres se convierte en una trasgresión a los órdenes de su propio género: 
Yo colaboraba con una colega, teníamos muchísimos años de trabajar juntas, éramos complementarias y, de repente, me empecé a dar cuenta que ella empezó a publicar las cosas sin que yo me diera cuenta. Cuando la confronto, pues me doy cuenta que muchas cosas de las que yo hacía, ella ya las había empezado a hacer y a copiar, también me pasó con otra colega de la UNAM (IE4).

Cuando por fin pasó un proyecto muy importante a CONACYT, se tuvo que resolver en un comité internacional, porque aquí en México se denunciaron "practicas misóginas" en la evaluación, ya cuando me lo aceptaron en ese comité internacional, una colega me dijo "tu único mérito es no tener hijos, por eso lo aprobaron" (IE2).

He tenido muy malas experiencias con colegas y, curiosamente, un par han sido mujeres, y que uno considera que son mis colegas, mis amigas, bueno, no amigas, pero cercanas, es tanta la competitividad que te roban las ideas, te roban el trabajo que estás haciendo [...]. La relación entre mujeres no es lo idílico que uno pensaría, "ay sí, somos mujeres y nos vamos a apoyar" (IE4).

Según los hallazgos de esta investigación, en el espacio laboral las barreras por razón de género aparecen en un proceso de reconfiguración debido, por un lado, a la fuerte demanda laboral y a la gran competitividad que existe entre el recurso humano altamente calificado con independencia del género al que pertenecen, pero con repercusiones más pronunciadas en el caso de las mujeres; y por otro lado, esa competitividad y la escasez de posiciones, aunado a la conciencia cada vez mayor de las mujeres sobre sus desventajas sociales y laborales, hace que ellas emprendan una lucha de poder, pero no contra los hombres sino contra las de su mismo género; las otras mujeres, que también aspiran a ocupar espacios laborales o mejores puestos, así como posiciones que implican ejercicio de poder, buscando quitar del camino a otras con la misma calificación y, en apariencia, con las mismas oportunidades de acceso. Lo anterior da cuenta de que algunas mujeres - y también hombres-, en espacios de trabajo altamente competitivos como la ciencia, lejos de mostrar un apoyo intragénero, se ven como adversarias potenciales.

También se identificaron relatos en donde se visibiliza de manera predominante la presencia masculina en los espacios laborales, especialmente en los puestos de poder y de mayor jerarquía, así como el avance, "ir más allá", que se advierte cuando existe la posibilidad de una "alianza" con sus pares hombres:

En el campo profesional, digamos que el hecho de ser mujer no ha jugado determinantemente, según lo veo yo, pero en la realidad debe haber algo que sí juega, porque no somos muchas mujeres dedicadas a la ciencia. Ahí, en los ámbitos laborales donde me he desempeñado, soy la única mujer y los demás son puros hombres, no es que te metan el pie por ser mujer, pero me llama la atención por ser la única mujer. Ya cuando subes a las esferas de toma de decisiones, ya es diferente [...], ahí ya es diferente,

\section{Diốlo@os}


vuelve a ser un ambiente netamente de hombres [...]. En el nivel de direcciones de área, solo estoy yo de mujer (IE1).

El prejuicio de algunos creo que es fuerte. México desafortunadamente no ha dejado de ser un lugar difícil en que, pues, una mujer solita pueda dedicarse a la ciencia. Creo que, sin duda, la alianza con un investigador es muy importante para que uno pueda, como mujer, pueda ir más allá (IE5).

Las experiencias anteriores llevaron a las científicas a configurar y desarrollar habilidades nuevas que ellas mismas describieron como necesarias para desenvolverse en la ciencia y en espacios laborales, tales como la capacidad de hacer relaciones "clave", generar y cultivar vínculos positivos, habilidad para hablar concretamente (ir al punto), negociar y tener "carisma" (capacidad de contagiar a otros/as de nuestras ideas-liderazgo). En el desarrollo de sus investigaciones se identificó que todas las científicas participantes en este estudio trabajan en proyectos de frontera en el ámbito internacional y que inciden en el desarrollo del país; algunos de sus proyectos tienen que ver con el diagnóstico automático de fallas en sistemas dinámicos y de fugas en ductos que transportan diversos fluidos, como agua, petróleo y gas, con el fin de evitar desastres ambientales y pérdidas humanas; también desarrollan radiofármacos para la medicina nuclear; trabajan en la gestión de desechos radioactivos usando técnicas como las analíticas atómicas pioneras en su tipo, que aseguran su disposición final y la protección al medio ambiente; producen y caracterizan películas delgadas de moléculas conductoras y diseñan y sintetizan películas delgadas con potenciales aplicaciones en áreas de ahorro de energía; realizan investigaciones relativas a celdas de combustible aniónicas para el almacenamiento de energía, así como proyectos pioneros en física nuclear experimental, que pueden brindan importantes avances tecnológicos y científicos a México.

\section{A manera de conclusión}

Los hallazgos de esta investigación dan cuenta de que los principales desafíos que las mujeres atraviesan en su formación académica y en el campo laboral en la ciencia son, en primer lugar, la fuerte demanda académica de las carreras de ingeniería, física y matemáticas, que requieren de los/as que aspiran a incorporarse a las mismas; la posesión de ciertas fortalezas, como la lógica-matemática, los idiomas, así como una gran capacidad de trabajo acompañada de disciplina para el estudio; habilidades que en México aún no son suficientemente promovidas en el estudiantado desde los niveles de educación básica y media superior, especialmente en las niñas y adolescentes. Otro desafío que se identificó fue el "clima frío" y hostil, naturalizado en los espacios académicos pertenecientes a estas carreras, que permite indagar en el contexto en el que a menudo se cuestionan las habilidades intelectuales de las mujeres que incursionan en ellos, reproduciendo discursos y prácticas que las aíslan. 
La falta de apoyo familiar fue un tercer desafío, debido a las construcciones culturales aparejadas al género femenino, que abren una brecha entre ellas y las carreras que eligen, cuyo imaginario social corresponde al "universo masculino", así como las expectativas que las y los progenitores tienen respecto a las elecciones profesionales de sus hijos/hijas, lo que se traduce también en cargas culturales para las mujeres que pueden afectar su elección de carrera.

En el campo laboral a su retorno a México, identificamos que el mayor desafío fue la falta de vínculos profesionales e incluso personales, debido a las rupturas que suelen producirse con la movilidad a un país extranjero para estudiar el posgrado. Una vez que se integran en los espacios laborales, los desafíos más recurrentes - pero no los únicos - fueron las barreras por razón de género, visibilizados en un proceso de reconfiguración debido, por un lado, a la fuerte demanda laboral en las instituciones de educación superior en México y, por el otro, a la gran competitividad existente entre el recurso humano altamente calificado - con independencia del género al que pertenecen-, que convergen con la falta de espacios laborales y crisis financiera, aunado a la conciencia cada vez mayor de las mujeres sobre sus desventajas sociales y laborales. Todo ello genera tensiones entre las investigadoras, e incluso prácticas desleales para quitar del camino a competidoras potenciales, lo que se puede traducir en una falta de apoyo intragénero.

Con respecto a las motivaciones, se identificó que principalmente, en retrospectiva, fungió como tal la imagen de sus referentes académicos positivos (mujeres y hombres) que atravesaron su experiencia durante la infancia y la adolescencia. La proyección de futuro que construyeron, con base en sus referentes académicos, fue, en primera instancia, lo que las movilizó a la acción para elegir una carrera científica. Las estrategias de avance fueron también diversas: emergió como fundamental la habilidad para actuar a su favor en el terreno profesional, lo que implicó la ruptura de ciertas reglas para generar sus propias oportunidades de acceso y desarrollo. Asimismo, en todas las participantes se identificó la capacidad de tomar decisiones por sí mismas, sin constreñirse a mandatos sociales bajo los cuales se enmarca al imaginario femenino, cuestión que desde este espacio impulsamos para que este tipo de experiencias sean tomadas en cuenta en las políticas de fortalecimiento de las vocaciones en las ciencias, desde una perspectiva de género.

\section{Referencias}

Archundia, E. (2020). México: avatares en el financiamiento a la ciencia y la tecnología. Revista de Psicología de la Universidad Autónoma del Estado de México, 9(18), 104-134. https://revistapsicologia.uaemex.mx/article/view/15584

Arredondo, T.; P. Vázquez; S. Velázquez (2019). STEM y brecha de género en Latinoamérica. Revista de El Colegio de San Luis, 8(18), 137-158. https://www.redalyc.org/jatsRepo/4262/426259447006/html/index.html 
Basail, A. (2020). Academias asediadas: convicciones y conveniencias ante la precarización. Buenos Aires: CLACSO.

Blazquez, N. (2011). El retorno de las brujas. Incorporación, aportaciones y críticas de las mujeres a la ciencia. México: UNAM.

Blazquez. N.; L. Fernández (2017). Política de ciencia y tecnología con perspectiva de género en México. Cadernos Pagu, (49), 1-32. https://www.scielo.br/scielo.php?pid=S0104833320170 $00100305 \&$ script $=$ sci arttext\&tlng $=$ es

Buquet, C.; J. Cooper; A. Mingo; H. Moreno (2013). Intrusas en la universidad. México: UNAM.

Bustos, O. (2002). Mujeres rompiendo el techo de cristal: el caso de las universidades. Revista de la Coordinación de Estudios de Posgrado (41), 1-7.

Bustos, O. (2008). Los retos de la equidad de género en la educación superior en México y la inserción de mujeres en el mercado laboral. Arbor, 184(733), 795-815. http://arbor.revistas. csic.es/index.php/arbor/article/view/225

Bustos, O. (2012). Mujeres en la educación superior, la academia y la ciencia. Ciencia, 63(3), 2433. https://www.ses.unam.mx/curso2013/pdf/Bustos Mujeres Educ Superior.pdf

Camarena, A.; G. Saavedra (2018). El techo de cristal en México. La Ventana, 15(47), 312-347. http://www.scielo.org.mx/pdf/laven/v5n47/1405-9436-laven-5-47-00312.pdf

Canales, A. (2011). La política científica y tecnológica en México. El impulso contingente en el periodo 1982-2006. México: Porrúa, UNAM.

Cervera, C.; V. Upegui (2018). Techo de cristal y suelo pegajoso: estudios de género en la academia. Verano de la Investigación Científica, 4(1), 1844-1848. http://www.jovenesenlaciencia.ugto.mx/index.php/jovenesenlaciencia/article/view/2886/2149

De Garay, A.; D. Del Valle (2012). Una mirada a la presencia de las mujeres en la educación superior en México. Revista Iberoamericana de Educación Superior, 6(3), 3-30. http://www.scielo. org.mx/pdf/ries/v3n6/v3n6a1.pdf

Dides, C.; M. Benavente; J. Morán (2008). Informe final. Estudio sobre formación de doctorados bajo el marco del PMG Sistema Enfoque de Género para CONICyT. Santiago: FLACSO. https:// www.conicyt.cl/wp-content/uploads/2015/03/Estudio-Sobre-Formacion-de-Doctorados-bajo-el-marco-del-PMG-Sistema-Enfoque-de-Genero-Diciembre-2008.pdf

García, A. (2016). Las docentes e investigadoras de la carrera de Física: impulsoras en el interés científico de las y los estudiantes. En Guevara. E.; A. García (coords.). Académicas que inspiran vocaciones científicas. La mirada de sus estudiantes. México: CEIICH, UNAM, 139-164.

García, G. (2005). Género, educación y política pública. La Ventana, 3(21), 70-89. http://www. scielo.org.mx/pdf/laven/v3n21/1405-9436-laven-3-21-70.pdf

Guevara, R.; C. Flores (2018). Educación científica de las niñas, vocaciones científicas e identidades femeninas. Experiencias de estudiantes universitarias. Revista Actualidades Investigativas en Educación, 18(2), 1-31. 
Hernández, O.; R. Pérez; G. González (2014). La deserción en los posgrados, un problema no menor. Diálogos sobre Educación. Temas actuales de investigación educativa, (8), 1-18. http:// dialogossobreeducacion.cucsh.udg.mx/index.php/DSE/article/view/309

Izquierdo, I. (2009). Mujeres en la academia ¿Cambio de oportunidades? Revista de Investigación y Divulgación sobre los Estudios de Género, (4), 51-66.

Izquierdo, I.; A. Atristan (2019). Experiencias de investigadoras en su ingreso, promoción y permanencia en el Sistema Nacional de Investigadores: tensiones y estrategias identitarias. Revista de Investigación Educativa, 10(18), 127-142.

Izquierdo, I.; N. Cárdenas (2019). Motivaciones, expectativas y medios de estudiantes cubanos y colombianos para realizar un posgrado en México. Revista Latinoamericana de Estudios Educativos, Colombia, 15(2), 111-133.

Kastersztein, J. (1999). Les stratégies identitaries des acteurs sociaux: aproche dynamique des finalités. En Camilleri. C.; J. Kastersztein; E. Lipiansky; H. Malewska-Peyre; I. Taboada-Leonetti; A. Vasquez (comps.). Stratégies identitaires. París: Presses Universitaires de France, 27-41.

Lomnitz, L.; M. Morán (1976). Estudio de mexicanos graduados en el extranjero. Ciencia y Desarrollo, (11), 45-50.

Mendoza, R. (2016). Las académicas ingenieras y su papel en el impulso a la investigación entre sus estudiantes. En Guevara. E.; A. García (coords.). Académicas que inspiran vocaciones científicas. La mirada de sus estudiantes. México: CEIICH, UNAM, 165-196.

Naranjo, P. (2004). Enfoques conductistas, cognitivos y racional emotivos. Costa Rica: Universidad de Costa Rica.

Naranjo, P. (2009). Motivación: Persectivas teóricas y algunas consideraciones de su importancia en el ámbito educativo. Revista Educación, 33(2), 153-170. https://www.redalyc.org/ pdf/440/44012058010.pdf

Pujadas, M. (1992). El método biográfico: el uso de las historias de vida en ciencias sociales. Madrid: Centro de Investigaciones sociológicas.

Quintana, D.; N. Blazquez (2017) Equidad de género en educación superior y ciencia. Agendas para América Latina y el Caribe. México: UNAM.

Ramos, M.; V. Sieglin; M. Zúñiga (2013). La intransparente transparencia. La asignación de sueldos y niveles académicos al profesorado en una universidad del norte de México. Perfiles Educativos, 35(141), 26-45.

Razo, G. (2008). La inserción de las mujeres en las carreras de ingeniería y tecnología. Perfiles Educativos, 30(121), 63-96. http://www.scielo.org.mx/scielo.php?script=sci arttext\&pid=S0185-26982008000300004\&lng=es\&tIng=es

Reis, B.; S. Cecílio (2014). Precarização, trabalho docente intensificado e saúde de professores universitários. Trabalho \& Educação, 23(2), 109-128.

Sieglin, V. (2012). El "techo de cristal" y el acoso laboral. Revista Ciencia, 63(3), 16-23. https:// www.revistaciencia.amc.edu.mx/index.php/ediciones-anteriores?id=201 
Valles, M. (2002). Entrevistas cualitativas. Madrid: Centro de Investigaciones Sociológicas.

Victorino, L.; R. Atriano; H. Rueda (2014). Políticas universitarias y neoliberalismo. Resultados y tendencias en la universidad latinoamericana. Diálogos sobre Educación. Temas actuales de investigación educativa, (9), 1-24. http://dialogossobreeducacion.cucsh.udg.mx/index.php/ DSE/article/view/294

Zubieta, G.; N. Marrero (2005). Participación de la mujer en la educación superior y la ciencia en México. Agricultura, Sociedad y Desarrollo, 2(1), 15-28. http://www.scielo.org.mx/pdf/asd/ v2n1/v2n1a2.pdf 